\title{
Roughness in surface growth equations
}

\author{
DIRK BLÖMKER ${ }^{\dagger}$ \\ Institut für Mathematik, RWTH Aachen, 52062 Aachen, Germany \\ STANISLAUS MAIER-PAAPE ${ }^{\dagger}$ \\ NWF I - Mathematik, RWTH Aachen, 52062 Aachen, Germany
}

AND

THOMAS WANNER ${ }^{\S}$

Department of Mathematics and Statistics, University of Maryland, Baltimore County, Baltimore, MD 21250, USA

[Received 9 August 2000 and in revised form 21 February 2001]

\begin{abstract}
We consider the roughness of surfaces described by stochastic partial differential equations on bounded domains which arise in surface growth equations. The roughness is usually described by the mean interface width, which is the expected value of the squared $L^{2}$-norm. Our main results describe the growth of the mean interface width for linear stochastic partial differential equations perturbed by white or colored noise.
\end{abstract}

Keywords: Surface roughness; molecular beam epitaxy; stochastic partial differential equations; additive white and colored noise; mean interface width.

\section{Introduction}

Surface growth problems study the spatio-temporal evolution of a surface profile generated through a growth process. These processes are not stationary, since the growth of the surface is due to constant deposition of material, as depicted in Fig. 1. One particular example is molecular beam epitaxy (MBE), where amorphous or crystalline material is deposited by a molecular beam onto an initially flat surface. Generally, the molecular beam is perturbed by stochastic fluctuations, such as, for example, Brownian motion in a gas - and therefore the process is expected to generate rather rough surfaces. On the other hand, there are several mechanisms that allow the particles to move on the surface while it is generated. One such process is surface diffusion, and it usually tends to smooth the surface. In light of these competing effects, one of the main problems consists in determining the actual roughness of the surface. Surveys on surface growth processes and molecular beam epitaxy can be found in Krug and Spohn [13], Meakin [16], Barabasi and Stanley [1], Halpin-Healy and Zhang [8], Marsili et al. [15], or Krug [12], to name just a few.

We consider the roughness of a surface described by the height function $h(t, x)$ at time $t \geqslant 0$ over some bounded domain $G \subset \mathbb{R}^{d}$. We assume that the temporal evolution of the height function

\footnotetext{
†Email: bloemker@instmath.rwth-aachen.de

*Email: maier@instmath.rwth-aachen.de

${ }^{\S}$ Email: wanner@math.umbc.edu
} 


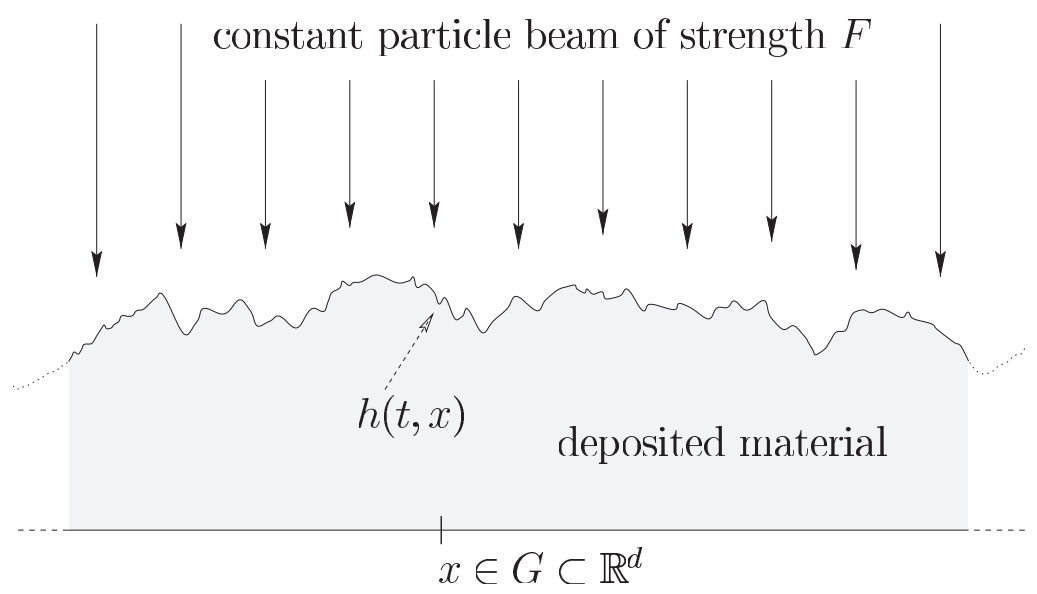

FIG. 1. Surface growth through material deposition.

is governed by a stochastic partial differential equation in $L^{2}(G)$ of the abstract form

$$
h_{t}(t)+A h(t)=F(h)(t)+\xi(t) \quad \text { for } t>0, \quad h(0)=0 .
$$

Here $A$ denotes the linear part, and $F$ the nonlinear part of the equation. In this paper we focus on $F \equiv 0$; the case of nonzero $F$ will be the topic of a forthcoming paper [3]. The stochastic term is given by a noise process $\xi=\{\xi(t)\}_{t} \geqslant 0$ which is the generalized derivative of a Wiener process in Hilbert space. Several specific models which fit the abstract form (1) have been proposed in the physics literature, such as the following models arising in surface growth and molecular beam epitaxy:

$$
\begin{aligned}
\text { Edwards, Wilkinson (1981) } & h_{t}=\Delta h+\xi \\
\text { Wolf, Villain (1990) } & h_{t}=-\Delta^{2} h+\xi \\
\text { Kadar, Parisi, Zhang (1986) } & h_{t}=\Delta h+|\nabla h|^{2}+\xi .
\end{aligned}
$$

For more details, we refer the reader to Edwards and Wilkinson [6], Wolf and Villain [20], and Kadar et al. [11]. All of the above equations are considered on cubes $[0, L]^{d}$ subject to periodic boundary conditions, and with (deterministic) initial value 0 . For more realistic nonlinear models for MBE see, for example, Hunt et al. [9, 10], Siegert and Plischke [19] and Raible et al. [18].

Roughness is physically defined through the scaling of the mean interface width $w(t)$ with respect to the length scale $L$ given by the underlying domain $G$. For this, the mean interface width $w(t)$ is defined by

$$
w(t):=\sqrt{\left\langle(h(t)-\bar{h}(t))^{2}\right\rangle} \text { where } \quad \bar{h}(t):=\frac{1}{|G|} \cdot \int_{G} h(t, x) \mathrm{d} x .
$$

See, for example, [15]. The brackets $\langle\cdot\rangle:=|G|^{-1} \cdot E \int_{G} \cdot \mathrm{d} x$ denote the mean value with respect to both space and probability. This notation is the usual physical notation, but it might be confused with the standard notation for scalar products in Hilbert spaces. Therefore, we will not use the above notation in this paper. 


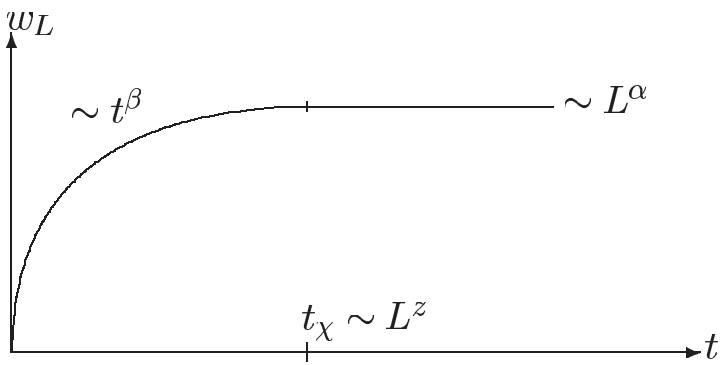

FIG. 2. Expected evolution of the mean interface width $w_{L}$.

As mentioned before, one is interested in understanding the temporal evolution of the mean interface width $w(t)$, as well as how this evolution relates to the underlying domain length scale $L$. Thus, one considers equation (1) on a family of domains $\{G(L)\}_{L>0}$, and studies the corresponding mean interface widths $w_{L}(t)$. Here $L$ is some measure for the size of $G(L)$.

Physical considerations show that the graph of the mean interface width $w_{L}$ is given qualitatively as depicted in Fig. 2. See, for example, the discussion in [15: p. 967]. More precisely, one expects the asymptotics

$$
w_{L}(t) \sim \begin{cases}t^{\beta} & \text { for } t<t_{\chi} \\ L^{\alpha} & \text { for } t>t_{\chi} .\end{cases}
$$

The exponent $\alpha$ is called the roughness exponent, and the growth exponent is given by $\beta$. Furthermore, the time $t_{\chi} \sim L^{z}$ at which the behavior of $w_{L}$ changes qualitatively is called the cross-over time with dynamic exponent $z$.

These exponents are usually related to each other by assuming that the model obeys the FamilyVicsek scaling relation $z=\alpha / \beta$. See, for example, [7]. The numerical values of the exponents are then typically derived by assuming self-affinity of the surface. In other words, one assumes that scaling the spatial variable $x$ to $l \cdot x$, the height variable $h$ to $l^{\alpha} \cdot h$, and time $t$ to $l^{z} \cdot t$ leaves the underlying equation statistically invariant. See Mandelbrot [14]. This determines the exponents $\alpha$ and $z$, and the missing exponent $\beta$ follows from the above scaling law. On the other hand, using $G=\mathbb{R}^{d}$ it is possible to employ Fourier transforms and a formal analysis to calculate the solution. From this the transient behavior of $w_{L}$ and the roughness exponent $\alpha$ can be determined. The values of the other exponents are then again consequences of additional laws.

The goal of this paper is to mathematically determine the temporal evolution of the mean interface width for a large class of stochastic partial differential equations of the form (1). We consider only linear stochastic partial differential equations, including the linear MBE models (2) by Edwards and Wilkinson and (3) by Wolf and Villain. Particular emphasis is given to the case of colored noise processes.

More precisely, the paper is organized as follows. After collecting basic properties of the mean interface width in Sections 2 and 3, the white noise case is addressed in Section 4. Here, we mathematically reproduce the temporal behavior indicated in Fig. 2 above. Section 5 deals with a class of colored noise processes corresponding to short-range correlations in the noise. Our main results for this case are contained in Theorems 5.3 and 5.4. They show that the temporal evolution of $w_{L}(t)$ depends crucially on the nature of the noise process and can differ significantly from the 
evolution indicated above. In many cases, we identify three different evolution regimes instead of two.

\section{Reductions and general setting}

This paper is devoted completely to the study of linear equations of the form

$$
h_{t}+A h=\xi, \quad h(0)=0,
$$

where $A$ is a linear operator on a suitable subspace of the Hilbert space $L^{2}(G)$, and the domain $G \subset \mathbb{R}^{d}$ describes the geometry of the base surface. In particular, this includes the molecular beam epitaxy models (2) by Edwards and Wilkinson, as well as (3) by Wolf and Villain. In the abstract equation (7), the inhomogeneity $\xi$ denotes an additive noise process. Depending on the regularity of $\xi$, one distinguishes between white noise, which is a space-time white noise process, and colored noise, which is white in time and spatially correlated. We treat these cases separately, concentrating on the white noise case in Section 4 and deferring the colored noise case to Section 5. First, however, we introduce some reductions in this section, which simplify our further studies. Moreover, we discuss basic properties of the mean interface width in Section 3, which are valid for both the white noise and the colored noise case.

As mentioned in the introduction, we are interested in describing the temporal evolution of the mean interface width $w$ of the surface $h$, whose evolution is described by (7). Recalling (5), we have

$$
w^{2}(t)=\frac{1}{|G|} \cdot E\|h(t)-\bar{h}(t)\|_{L^{2}(G)}^{2}
$$

where $\bar{h}(t)$ denotes the spatial average of the surface as defined in (5). In general, this average will grow in time due to the constant deposition of material. For the sake of simplifying the presentation, we want to assume without loss of generality that $\bar{h}(t)=0$ for all $t \geqslant 0$. This can of course be achieved easily by switching to the moving frame $\tilde{h}:=h-\bar{h}$, but renders a transformed evolution law for $\tilde{h}$. More precisely, the shifted surface $\tilde{h}$ formally satisfies an equation of the form

$$
\tilde{h}_{t}+A \tilde{h}=(\xi-\bar{\xi})+(\overline{A h}-A \bar{h}), \quad \tilde{h}(0)=0 .
$$

The term in the first parentheses is to be expected, since it just corresponds to considering the stochastic noise process in a moving frame, i.e. to assuming that the spatial average of the noise vanishes. However, in order to arrive at an equation of the form given in (7), the term in the second parentheses has to vanish. This is equivalent to requiring that computing the spatial average commutes with the linear operator $A$. Fortunately, this is satisfied in many situations of interest, in particular in the linear MBE models (2) and (3). More generally, it is satisfied if $A$ is a selfadjoint operator with closed range, whose nullspace contains the constant functions. In this case, the commutativity follows from the closed-range theorem.

In order to keep our presentation as simple as possible, we assume in the following that it is possible to assume $\bar{h}(t)=0$ for all $t \geqslant 0$ without loss of generality, i.e. that the operator $A$ exhibits the above-mentioned commutativity.

\section{Basic properties}

We begin our discussion of the linear growth equation (7) by collecting the basic assumptions on the linear operator $A$ and the noise process $\xi$. We also derive a few properties of the mean interface width $w$ defined in (5), which apply to both the white noise and the colored noise case. 
As mentioned in the previous section, we assume without loss of generality that the solution $h$ of (7) satisfies $\bar{h}(t)=0$ for all $t \geqslant 0$. Thus, we consider (7) as an abstract evolution equation on the space

$$
X:=\left\{h \in L^{2}(G): \int_{G} h(x) \mathrm{d} x=0\right\}
$$

where $G \subset \mathbb{R}^{d}$ denotes a bounded domain with sufficiently smooth boundary. The precise assumptions on $G$ will be specified in more detail later. We begin by specifying the assumptions on the linear operator $A$ and the noise process $\xi$.

Assumption 3.1 Let $G \subset \mathbb{R}^{d}$ be a bounded domain, and consider the Hilbert space $X$ defined in (10). Let $A: X \rightarrow X$ denote a self-adjoint and sectorial linear operator. Assume that there exists an orthonormal basis $\left\{f_{k}\right\}_{k \in \mathbb{N}}$ of $X \subset L^{2}(G)$ which consists of eigenfunctions of $A$, and that the corresponding ordered real eigenvalues $\left\{\lambda_{k}\right\}_{k \in \mathbb{N}}=\left\{\lambda_{k}(G)\right\}_{k \in \mathbb{N}}$ satisfy $\lambda_{k} \rightarrow \infty$ as $k \rightarrow \infty$. Moreover, let $\left\{\alpha_{k}\right\}_{k \in \mathbb{N}}$ denote a sequence of non-negative real numbers such that

$$
\sum_{k=k_{o}}^{\infty} \frac{\alpha_{k}^{2}}{\lambda_{k}}<\infty \quad \text { for some } \quad k_{o} \in \mathbb{N} .
$$

The noise process $\xi$ is modeled as the generalized derivative of the $Q$-Wiener process

$$
W(t)=\sum_{k \in \mathbb{N}} \alpha_{k} \cdot \beta_{k}(t) \cdot f_{k} \text { for } t \geqslant 0
$$

where $\left\{\beta_{k}\right\}_{k \in \mathbb{N}}$ denotes a family of independent standard Brownian motions over a common probability space $(\Omega, \mathcal{A}, P)$.

If in the above assumption we have $\alpha_{k}=$ const. for all $k \in \mathbb{N}$, then the noise is referred to as white noise. In all other cases it is called colored noise. For more details on space-time white noise (sometimes also referred to as cylindrical noise) and colored noise we refer the reader to Chapter 4 in [5].

In the physics literature the colored noise we consider is referred to as spatially correlated noise, since the noise process is still white (or uncorrelated) in time. For results on the influence of correlations in time see, for example, [17], where the authors establish results for the KPZ equation (4) on the whole real line with long-range correlations in the noise by using a renormalization group analysis.

Under the above assumptions, it is well known that (7) has a unique weak solution in $X \subset L^{2}(G)$. This solution is given explicitly by the stochastic convolution $W_{A}$ defined as

$$
W_{A}(t)=\sum_{k \in \mathbb{N}} \alpha_{k} \cdot \int_{0}^{t} \mathrm{e}^{-(t-\tau) \lambda_{k}} \mathrm{~d} \beta_{k}(\tau) \cdot f_{k} \in X
$$

where all stochastic integrals are in the sense of Ito. In other words, we have an infinite-dimensional Ornstein-Uhlenbeck process $\left\{W_{A}(t)\right\}_{t} \geqslant 0$ without drift, where $W_{A}(t)$ is normally distributed in $L^{2}(G)$ with mean 0 and covariance operator $Q \cdot \int_{0}^{t} \mathrm{e}^{-2 \tau A} \mathrm{~d} \tau$. Here $Q$ denotes the covariance operator of $W$, i.e. we have $Q f_{k}=\alpha_{k}^{2} f_{k}$. 
For any $t \geqslant 0$ the series in (13) converges in $L^{2}\left(\Omega, L^{2}(G)\right)$, which is the Hilbert space consisting of all $L^{2}(G)$-valued random variables on $(\Omega, \mathcal{A}, P)$ with finite second moment, equipped with the norm $\left(E\|\cdot\|_{L^{2}(G)}^{2}\right)^{1 / 2}$. See, for example, Theorem 5.4 in [5]. In other words, $h(t)=$ $W_{A}(t) \in X$ describes the surface of the growth process at time $t$. Notice also that the formulation of (7) in $X$ automatically implies the validity of $\bar{h}(t)=0$ for all $t \geqslant 0$. As we mentioned at the end of the last section, this can be assumed without loss of generality in most cases of interest, such as the ones discussed in the following example.

EXAMPLE 3.2 Let $\tilde{p}: \mathbb{R} \rightarrow \mathbb{R}$ be a polynomial with $\tilde{p}(0)=0$ and $\tilde{p}(x) \rightarrow \infty$ for $x \rightarrow \infty$. Moreover, assume that $G=[0, L]^{d}$ for some fixed $L>0$, and let $\Delta_{\text {per }}$ denote the Laplacian on $G$ subject to periodic boundary conditions. Then the operator $A=\tilde{p}\left(-\Delta_{\text {per }}\right)$ satisfies all the requirements contained in Assumption 3.1. Notice also that $\tilde{p}\left(-\Delta_{\text {per }}\right)$ is self-adjoint on $L^{2}(G)$ and maps constant functions to zero. Thus, assuming $\bar{h}(t) \equiv 0$ is without loss of generality for examples of this type. For more details, compare the discussion at the end of the last section.

Of particular interest are the cases $\tilde{p}(x)=x$ and $\tilde{p}(x)=x^{2}$, since they correspond to the MBE models (2) and (3), respectively.

In the following, we will mainly concentrate on examples of the above type. However, other situations are of interest as well. For example, let $G \subset \mathbb{R}^{d}$ denote a bounded domain with sufficiently smooth boundary, let $\tilde{p}$ be as above, and consider $A=\tilde{p}(-\Delta)$ on $G$ subject to homogeneous Neumann boundary conditions. Then Assumption 3.1 is satisfied as well.

As mentioned in the introduction, we are interested in describing the temporal evolution of the mean interface width $w$ of the surface $h$ as defined in (5). Due to $h(t) \in X \subset L^{2}(G)$, the mean interface width is well defined for the unique weak solution $h(t)=W_{A}(t)$ of (7). Notice, however, that the surface $h$ does not necessarily exhibit regularity higher than $L^{2}(G)$. Nevertheless, for physical applications, it seems reasonable to require that the surface is at least continuous. In other words, one is interested in versions of the stochastic convolution $h(t)=$ $W_{A}(t)$ which are continuous with respect to the spatial variable. Questions of this type can be settled using the regularity theory of Section 5.5 in [5], which is based on the celebrated Kolmogorov test. Specifically, Theorem 5.20 in [5] implies the existence of a continuous version of $W_{A}$ in $C^{0}([0, T] \times G)$, provided the eigenfunctions $f_{k}$ and the eigenvalues $\lambda_{k}$ satisfy certain conditions. These conditions are basically growth conditions for the $\lambda_{k}$, as well as $L^{\infty}$-bounds on the eigenfunctions $f_{k}$ and their gradients, i.e. they can be viewed as geometric conditions for the domain $G$. Fortunately, these conditions are automatically satisfied in the situation of Example 3.2. In general, however, the existence of a continuous version of the weak solution $h(t)=W_{A}(t)$ depends crucially on the specific shape of the domain $G$.

For the remainder of this section we deduce some first properties of the mean interface width, assuming that Assumption 3.1 is satisfied. In this case, we have

$$
w^{2}(t)=\frac{1}{|G|} \cdot E\|h(t)-\bar{h}(t)\|_{L^{2}(G)}^{2}=\frac{1}{|G|} \cdot E\left\|W_{A}(t)\right\|_{X}^{2} .
$$

Using the structure of the noise process as required in Assumption 3.1, the Ito-isometry immediately implies the following explicit representation of the mean interface width, provided all eigenvalues $\lambda_{k}$ are nonzero:

$$
w^{2}(t)=\frac{1}{|G|} \cdot \sum_{k \in \mathbb{N}} \alpha_{k}^{2} \cdot \int_{0}^{t} \mathrm{e}^{-2(t-\tau) \lambda_{k}} \mathrm{~d} \tau=\frac{1}{2|G|} \cdot \sum_{k \in \mathbb{N}} \frac{\alpha_{k}^{2} \cdot\left(1-\mathrm{e}^{-2 t \lambda_{k}}\right)}{\lambda_{k}} .
$$


If on the other hand $\lambda_{k}=0$ for some $k \in \mathbb{N}$, then the corresponding term in the last series of (14) equals $\alpha_{k}^{2} \cdot t$. Notice that the convergence of the series follows automatically from Assumption 3.1. The following lemma contains some elementary, yet useful properties of $w$ which can be derived easily using the above representation.

LEMMA 3.3 Assume that Assumption 3.1 is satisfied, and consider the square $w^{2}(t)$ of the mean interface width as given in (14). Then the following holds:

(a) The function $w^{2}$ is in $C^{\infty}(0, \infty)$, and for $t>0$ and $n \in \mathbb{N}$ we have

$$
\frac{\mathrm{d}^{n}}{\mathrm{~d} t^{n}} w^{2}(t)=\frac{1}{|G|} \cdot \sum_{k \in \mathbb{N}} \alpha_{k}^{2} \cdot\left(-2 \lambda_{k}\right)^{n-1} \cdot \mathrm{e}^{-2 t \lambda_{k}} .
$$

Thus, the mean interface width $w$ is infinitely many times differentiable for $t>0$.

(b) Both $w^{2}$ and $w$ are strictly increasing for $t \geqslant 0$. If in addition $\lambda_{1}>0$, then $w^{2}$ is concave and its derivatives have alternating signs.

(c) If the smallest eigenvalue $\lambda_{1}$ of the operator $A$ is strictly positive, then the limit $w^{2}(\infty):=$ $\lim _{t \rightarrow \infty} w^{2}(t)$ exists and is given by

$$
w^{2}(\infty)=\frac{1}{2|G|} \cdot \sum_{k \in \mathbb{N}} \frac{\alpha_{k}^{2}}{\lambda_{k}} .
$$

If $\lambda_{1}<0$, then $w^{2}(t) \sim \mathrm{e}^{-2 t \lambda_{1}}$ as $t \rightarrow \infty$, and in the case $\lambda_{1}=0$ one obtains $w^{2}(t) \sim t$.

Proof. Due to (11), the series $\sum_{k=k_{0}}^{\infty} \alpha_{k}^{2} / \lambda_{k}$ is absolutely convergent for some $k_{0} \in \mathbb{N}$. Furthermore, it is clear that for any $n \geqslant 1$ there exists a constant $C>0$ such that for all sufficiently large $k \in \mathbb{N}$ we have

$$
\left|\alpha_{k}^{2} \cdot\left(-2 \lambda_{k}\right)^{n-1} \cdot \mathrm{e}^{-2 t \lambda_{k}}\right| \leqslant \frac{C \cdot \alpha_{k}^{2}}{t^{n} \cdot \lambda_{k}} .
$$

Together, these observations immediately furnish the uniform convergence of the series $\sum_{k \in \mathbb{N}} \alpha_{k}^{2}$. $\left(-2 \lambda_{k}\right)^{n-1} \cdot \mathrm{e}^{-2 t \lambda_{k}}$ on $\left[t_{0}, \infty\right)$, for every $t_{0}>0$. A standard result from real analysis now implies that the $n$th derivative of $w^{2}(t)$ exists and is given as in (a). From this representation, the remaining assertions of the lemma follow easily.

Notice that the differentiability of $w^{2}$ at $t=0$ depends crucially on the precise asymptotic behavior of the eigenvalues $\lambda_{k}$ and the noise coefficients $\alpha_{k}$. Also, the fact that $w(t)$ grows without bound if $\lambda_{1} \leqslant 0$ does not make sense physically. Therefore, we will generally assume that $A$ is a strictly positive operator, i.e. that $\lambda_{1}>0$.

\section{Results for white noise}

In this section, we investigate the behavior of the mean interface width for the case of white noise, i.e. we assume $\alpha_{k}=1$ for all $k \in \mathbb{N}$ in Assumption 3.1. Lemma 3.3 provided some small first insight into the long-time asymptotic behavior of the mean interface width $w$ as $t \rightarrow \infty$. As outlined in the introduction, we are also interested in the behavior of $w$ for small $t>0$, i.e. the transient behavior of the mean interface width. We expect that the behavior in these two regimes is qualitatively as depicted in Fig. 2. In particular, we are interested in results showing how the different types of 
behavior can be quantified using a typical length scale $L$ associated with the domain $G$. In our examples (e.g. (2) or (3)) we had $G(L)=] 0, L{ }^{d}$. The general case is covered by the following assumption.

Assumption 4.1 Let $G(L)$ denote a one-parameter family of open and connected subsets of $\mathbb{R}^{d}$ which depend on a positive real scalar $L$. Suppose that there are $L$-independent positive constants $c_{G}^{\prime}, C_{G}^{\prime}, c_{G}$, and $C_{G}$ such that for all $L \in(0, \infty)$ the area and the diameter of $G(L)$ satisfy

$$
c_{G} \cdot L^{d} \leqslant|G(L)| \leqslant C_{G} \cdot L^{d} \quad \text { and } \quad c_{G}^{\prime} \cdot L \leqslant \operatorname{diam}(G(L)) \leqslant C_{G}^{\prime} \cdot L .
$$

In order to emphasize the dependence of the mean interface width $w$ on the scale parameter $L$, we write $w_{L}=w$ from now on.

Since we consider one-parameter families of domains $G(L)$ from now on, we also have to consider one-parameter families of corresponding linear operators $A(L)$, defined on appropriate subspaces of $L^{2}(G(L))$. In our applications, these operators are always a fixed differential operator $A$ acting on different domains $G(L)$ in the obvious way, subject to the same boundary conditions.

In the following, we mathematically derive the short-term and long-term dynamics of the mean interface width $w_{L}$ of the weak solution $h$ of (7). Specifically, we establish the validity of the asymptotics given in (6), i.e. we determine the exponents $\alpha, \beta$, and $z$. The remainder of this section is divided into three parts. We begin by determining the large-time behavior of $w_{L}$, then the transient behavior is estimated. For both of these steps we need additional assumptions. In the last part, we summarize and discuss our results.

\subsection{Long-time asymptotic behavior}

In order to obtain results relating the long-time asymptotics of $w_{L}$ (cf. Lemma 3.3) quantitatively to the length scale $L$, we need to impose further assumptions.

Assumption 4.2 Assume there exist an $m>0$ and two sequences $\left\{\hat{c}_{k}\right\}_{k \in \mathbb{N}}$ and $\left\{\hat{C}_{k}\right\}_{k \in \mathbb{N}}$ of positive constants such that $\sum_{k \in \mathbb{N}}\left(1 / \hat{c}_{k}\right)<\infty$, as well as

$$
\frac{\hat{c}_{k}}{L^{2 m}} \leqslant \lambda_{k}(L) \leqslant \frac{\hat{C}_{k}}{L^{2 m}} \quad \text { for all } \quad L>0, \quad k \in \mathbb{N},
$$

where $\lambda_{k}(L)$ denotes the $k$ th eigenvalue of $A$ on the domain $G(L)$ (cf. Assumption 3.1).

It can easily be verified that in the situation of Example 3.2 this condition is satisfied provided $\tilde{p}>0$ on $\mathbb{R}^{+}$and $\operatorname{deg} \tilde{p}=m$. Notice also that Assumption 4.2 automatically implies $\lambda_{k}(L)>0$ for all $k \in \mathbb{N}$.

Combining Assumptions 4.1 and 4.2 with Lemma 3.3, the long-time behavior of the mean interface width can be determined easily. Lemma 3.3(c) implies

$$
w_{L}^{2}(\infty)=\frac{1}{2|G|} \cdot \sum_{k \in \mathbb{N}} \frac{1}{\lambda_{k}(L)} \leqslant \frac{1}{2 c_{G}} \cdot L^{2 m-d} \cdot \sum_{k \in \mathbb{N}} \frac{1}{\hat{c}_{k}} .
$$

Analogously, one can easily obtain a lower bound on $w_{L}^{2}(\infty)$. This furnishes the validity of the long-time asymptotics in (6) with roughness exponent $\alpha$ given by

$$
\alpha=m-\frac{d}{2} .
$$


As for the absolute error between the squares of the actual value of the mean interface width $w_{L}(t)$ and the limit $w_{L}(\infty)$ one obtains

$$
\left|w_{L}^{2}(t)-w_{L}^{2}(\infty)\right|=\frac{1}{2|G|} \cdot \sum_{k \in \mathbb{N}} \frac{\mathrm{e}^{-2 t \lambda_{k}}}{\lambda_{k}}<\mathrm{e}^{-2 t \lambda_{1}} \cdot w_{L}^{2}(\infty) .
$$

Thus, the relative error $\left|w_{L}^{2}(t)-w_{L}^{2}(\infty)\right| / w_{L}^{2}(\infty)$ is smaller than a specified threshold $0<p \ll 1$, provided $\mathrm{e}^{-2 t \lambda_{1}} \leqslant p$. This finally yields

$$
t \geqslant-\frac{\ln p}{2 \lambda_{1}} \geqslant C_{p} \cdot L^{2 m}
$$

where $C_{p}:=-(\ln p) /\left(2 \hat{C}_{1}\right)$. This in turn furnishes a lower bound on the dynamic exponent $z$ defined in (6), namely $z \geqslant 2 m$.

\subsection{Transient behavior}

We now turn our attention to the transient behavior of the mean interface width. This is accomplished basically by approximating the sums in (14) by integrals, and then by extracting the $t$-dependence from the integrals through an appropriate substitution. However, this can only be achieved under sharper assumptions on the eigenvalues $\lambda_{k}(L)$. More precisely, we need the following sharpening of Assumption 4.2.

Assumption 4.3 Assume there are strictly positive constants $n, c_{A}$, and $C_{A}$ such that

$$
c_{A} \cdot \frac{k^{2 n}}{L^{2 m}} \leqslant \lambda_{k}(L) \leqslant C_{A} \cdot \frac{k^{2 n}}{L^{2 m}} \quad \text { for all } \quad L>0, \quad k \in \mathbb{N} .
$$

Notice that the additional condition $n>1 / 2$ is necessary in order to make sure that Assumption 4.3 implies Assumption 4.2. Furthermore, it is needed to guarantee the validity of (11) in Assumption 3.1.

In most cases of interest, the above condition on the asymptotic behavior of the eigenvalues is automatically satisfied. If we assume $\tilde{p}(x)=x^{m}$ in Example 3.2, then all the eigenvalues are known explicitly, and it can easily be verified that (17) holds with $n=m / d$. More generally, if $G(L)=L \cdot G_{0}$, then the necessary asymptotics of the eigenvalues follows from a standard result in Courant and Hilbert [4], in combination with a simple scaling argument. Also in this case we have $n=m / d$.

We begin with some technical estimates for the terms in (14). Using Assumption 4.3, one obtains

$$
\underbrace{\frac{1-\mathrm{e}^{-2 c_{A} k^{2 n} L^{-2 m} t}}{C_{A} k^{2 n}} \cdot L^{2 m}}_{=: I_{1}(k, L, t)} \leqslant \frac{1-\mathrm{e}^{-2 t \lambda_{k}(L)}}{\lambda_{k}(L)} \leqslant \underbrace{\frac{1-\mathrm{e}^{-2 C_{A} k^{2 n} L^{-2 m} t}}{c_{A} k^{2 n}} \cdot L^{2 m}}_{=: I_{2}(k, L, t)} .
$$

Due to $\lim _{k \rightarrow 0} I_{1}(k, L, t)=2 t c_{A} / C_{A}$ and the fact that $I_{1}(\cdot, L, t)$ and $I_{2}(\cdot, L, t)$ are decreasing on $\mathbb{R}^{+}$, for all $t>0$ and $L>0$, a standard argument using Riemann sums implies

$$
\int_{0}^{\infty} I_{1}(\kappa, L, t) \mathrm{d} \kappa-2 t c_{A} C_{A}^{-1} \leqslant \sum_{k \in \mathbb{N}} \frac{1-\mathrm{e}^{-2 t \lambda_{k}(L)}}{\lambda_{k}(L)} \leqslant \int_{0}^{\infty} I_{2}(\kappa, L, t) \mathrm{d} \kappa .
$$


Applying the change of variables $\tilde{\kappa}=\left(c_{A} \cdot t\right)^{1 /(2 n)} \cdot L^{-m / n} \cdot \kappa$ to the integral on the left-hand side, and assuming that $n>1 / 2$ in Assumption 4.3, furnishes the identity

$$
\int_{0}^{\infty} I_{1}(\kappa, L, t) \mathrm{d} \kappa=K \cdot c_{A}^{1-1 /(2 n)} \cdot C_{A}^{-1} \cdot L^{m / n} \cdot t^{1-1 /(2 n)}
$$

where the constant $K$ depends only on $n$. Analogously, one can show that exchanging $c_{A}$ and $C_{A}$ in the right-hand side of (19) yields an explicit formula for $\int_{0}^{\infty} I_{2}(\kappa, L, t) \mathrm{d} \kappa$. Finally, (14), (18), and Assumption 4.1, in combination with (19) and its analogue for $I_{2}$ imply

$$
C_{1} \cdot t^{1-1 /(2 n)} \cdot L^{m / n-d}-C^{\prime} \cdot t \cdot L^{-d} \leqslant w_{L}^{2}(t) \leqslant C_{2} \cdot t^{1-1 /(2 n)} \cdot L^{m / n-d}
$$

where the constants $C_{1}, C_{2}$, and $C^{\prime}$ are given by $C_{1}:=K c_{A}^{1-1 /(2 n)} /\left(2 C_{A} C_{G}\right), C_{2}:=K C_{A}^{1-1 /(2 n)} /$ $\left(2 c_{A} c_{G}\right)$, and $C^{\prime}:=c_{A} /\left(C_{A} c_{G}\right)$.

It is clear from (20) that in general the transient behavior of $w_{L}(t)$ depends on the scaling factor $L$. However, in most cases of interest, such as Example 3.2 with $\tilde{p}(x)=x^{m}$, we have $n=m / d$, i.e. the $L$-dependence disappears in the leading terms of (20). In this case, the transient growth of the mean interface width $w_{L}(t)$ is as suggested by (6), and the growth exponent $\beta$ is given by

$$
\beta=\frac{1}{2}-\frac{d}{4 m}
$$

In order to make sure that the squared mean interface width $w_{L}^{2}(t)$ stays sufficiently close to the channel bounded by the curves $C_{i} \cdot L^{m / n-d} \cdot t^{1-1 /(2 n)}$ for $i=1$, 2, we have to control its relative distance to the channel, i.e. we have to bound

$$
\frac{C^{\prime} \cdot t \cdot L^{-d}}{C_{1} \cdot t^{1-1 /(2 n)} \cdot L^{m / n-d}}=\frac{C^{\prime}}{C_{1}} \cdot t^{1 /(2 n)} \cdot L^{-m / n}
$$

by some small number $0<p \ll 1$. This can obviously be achieved if we choose

$$
t \leqslant c_{p} \cdot L^{2 m}
$$

where $c_{p}$ denotes a suitable constant which is independent of $t$ and $L$, but does depend on $p, n$, and the constants $c_{A}, c_{G}, C_{A}$, and $C_{G}$. Together with (16) this finally implies

$$
t_{\chi}=t_{\chi}(L) \sim L^{2 m} \quad \text { and } \quad z=2 m .
$$

\subsection{Main results and discussion}

In the last two sections, we derived the desired statements describing the asymptotic behavior of the mean interface width $w_{L}(t)$ of the surface $h$ given by (7). These results can be summarized as follows.

THEOREM 4.4 Let $A$ denote a linear operator on the space $X$ defined in (10), and suppose that Assumptions 3.1, 4.1, and 4.3 hold with $n>1 / 2$. Furthermore, assume $\alpha_{k}=1$ for all $k \in \mathbb{N}$, i.e. that the noise is white noise. 


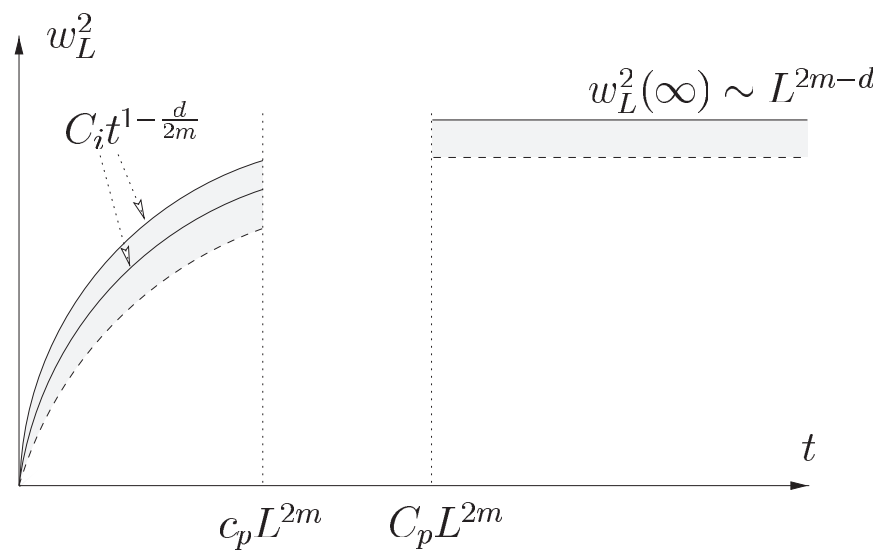

FIG. 3. Surface roughness with relative error $p>0$ for $A=\left(-\Delta_{\mathrm{per}}\right)^{m}$.

Then there are positive constants $C_{1}, \ldots, C_{4}$ which are independent of both $t$ and $L$ such that for any error bound $0<p \ll 1$ the following holds. There exist positive constants $c_{p}$ and $C_{p}$ which are independent of both $t$ and $L$ such that for all $L>0$ we have

$$
\begin{array}{rlrl}
w_{L}^{2}(t) & \in\left[C_{1}-p, C_{2}\right] \cdot t^{1-1 /(2 n)} \cdot L^{m / n-d} & \text { for } t \leqslant c_{p} \cdot L^{2 m}, \\
w_{L}^{2}(t) & \in[1-p, 1] \cdot w_{L}^{2}(\infty) & \text { for } t \geqslant C_{p} \cdot L^{2 m}, \\
w_{L}^{2}(\infty) & \in\left[C_{3}, C_{4}\right] & \cdot L^{2 m-d} . &
\end{array}
$$

Thus, the dynamic exponent $z$ and the roughness exponent $\alpha$ in (6) are given by $z=2 \mathrm{~m}$ and $\alpha=m-d / 2$. If we additionally assume $n=m / d$, then the growth exponent $\beta$ is given by $\beta=1 / 2-d /(4 m)$, in accordance with the Family-Vicsek scaling law $z=\alpha / \beta$.

This theorem applies to the case $A=\left(-\Delta_{\text {per }}\right)^{m}$ on $[0, L]^{d}$ with $n=m / d$, provided $2 m>d$. See also Example 3.2. In this case the result of Theorem 4.4 is sketched in Fig. 3. It mathematically reproduces known results from the physics literature, as described in (6) and derived by scaling arguments. To be more precise, we obtain the same exponents $\alpha, \beta$, and $z$. Due to Lemma 3.3 the squared mean interface width $w_{L}^{2}$ is monotone and convex, and therefore the graph of $w_{L}^{2}$ is qualitatively as given in Fig. 2.

Furthermore, in this case, all the eigenvalues are known explicitly, and therefore one can easily determine the constants $C_{1}, \ldots, C_{4}$ in Theorem 4.4. While we refrain from giving the specific values for these constants, we add a few comments on the one-dimensional case $d=1$. Here, the sequence of eigenvalues is given by $\lambda_{2 k-1}(L)=\lambda_{2 k}(L)=(2 k \pi / L)^{2 m}$ for $k \in \mathbb{N}$, i.e. all eigenvalues are double. Using this fact, one can show that the series on the right-hand side of (14) is twice the value of the sub-series in which $k$ ranges only over the even integers. This latter series can be estimated using the results of Sections 4.1 and 4.2, now with $c_{A}=C_{A}$. In addition, we immediately have $c_{G}=C_{G}$ in Assumption 4.1. From this, one can readily deduce that Theorem 4.4 holds with $C_{1}=C_{2}$.

REMARK 4.5 For mixed operators such as $A=\Delta_{\text {per }}^{2}-\Delta_{\text {per }}$ on $[0, L]^{d}$, our Assumptions 4.2 and 4.3 are not satisfied for all $L>0$. Nevertheless, using the techniques of the proof of Theorem 4.4 
one can still recover similar results. For example, if $A$ is a polynomial in $-\Delta_{\text {per }}$, then for $0<L \leqslant$ $L_{0}$ the asymptotic behavior of $w_{L}^{2}$ is determined by the highest occurring power of $-\Delta_{\text {per }}$.

Unfortunately, the situation is more complicated for large $L$. It is not too difficult to modify our results in such a way that estimates on the asymptotic behavior of $w_{L}^{2}$ can be derived. In general, however, the exponents $\alpha, \beta$, and $z$ are no longer well defined, and the transient behavior of the mean interface width depends on $L$.

\section{Results for colored noise}

In this section the results for space-time white noise are extended to spatially correlated noise. We consider colored noise which is generated by a $Q$-Wiener process as given in Assumption 3.1, but in order to keep our presentation as simple as possible we focus on the specific subclass described in the following assumption.

Assumption 5.1 Let $G(L) \subset \mathbb{R}^{d}$ denote a family of bounded domains and assume that for each $L$ Assumption 3.1 is satisfied. Furthermore, we assume that the following holds for the coefficients $\alpha_{k}(L)$ of the $Q$-Wiener processes $W$ defined in (12): there exist constants $c_{\Gamma}, C_{\Gamma}>0$ and $\gamma \geqslant 0$ such that

$$
\frac{c_{\Gamma}}{1+k^{\gamma} \cdot L^{-d \gamma}}<\alpha_{k}^{2}(L)<\frac{C_{\Gamma}}{1+k^{\gamma} \cdot L^{-d \gamma}}
$$

for all $k \in \mathbb{N}$ and $L>0$.

The inequality in (21) requires that the coefficients $\alpha_{k}(L)$ exhibit a well defined algebraic decay rate in the limit $k L^{-d} \rightarrow \infty$. This assumption is motivated by noise processes with constant shape covariance functional on large domains, such as, for example, with bounded correlation length of the noise, which was discussed for $d=1$ in [2]. Other types of asymptotic behavior of the coefficients $\alpha_{k}(L)$ can also be discussed, but the results depend crucially on the specific behavior. See, for example, Corollary 5.7 at the end of Section 5.3.

Our main results are stated and discussed in Section 5.3, but before beginning our discussion of the long-time behavior of the mean interface width, we collect a few assumptions and facts which are important for both the long-time and the transient behavior. We assume as before that the eigenvalues $\lambda_{k}(L)$ satisfy Assumption 4.3 with $n=m / d$, and that $G=G(L)$ satisfies Assumption 4.1. Then (11) is satisfied if and only if $\gamma+2 m / d>1$, which we therefore also assume from now on.

\subsection{Long-time asymptotic behavior}

In order to establish the long-time asymptotics of the mean interface width $w_{L}(t)$, we proceed in a similar way to Section 4.1. Equation (14) implies

$$
\begin{aligned}
& \frac{c_{\Gamma}}{2 C_{A} C_{G}} \cdot L^{2 m-d} \cdot \sum_{k \in \mathbb{N}} \frac{1-\mathrm{e}^{-2 c_{A} k^{2 m / d} L^{-2 m} t}}{k^{2 m / d} \cdot\left(k^{\gamma} L^{-d \gamma}+1\right)} \leqslant w_{L}^{2}(t) \\
& \leqslant \frac{C_{\Gamma}}{2 c_{A} c_{G}} \cdot L^{2 m-d} \cdot \sum_{k \in \mathbb{N}} \frac{1-\mathrm{e}^{-2 C_{A} k^{2 m / d} L^{-2 m} t}}{k^{2 m / d} \cdot\left(k^{\gamma} L^{-d \gamma}+1\right)},
\end{aligned}
$$


and therefore

$$
w_{L}^{2}(\infty) \in\left[\frac{c_{\Gamma}}{2 C_{A} C_{G}}, \frac{C_{\Gamma}}{2 c_{A} c_{G}}\right] \cdot L^{2 m-d} \cdot \sum_{k \in \mathbb{N}} \frac{1}{k^{2 m / d} \cdot\left(k^{\gamma} L^{-d \gamma}+1\right)}
$$

for all $L>0$. Notice that since $\gamma+2 m / d>1$, the series is finite and we can establish its asymptotic behavior.

Lemma 5.2 Consider $\vartheta, \gamma \geqslant 0$ with $\vartheta+\gamma>1$ and define

$$
S(x)=\sum_{k \in \mathbb{N}} \frac{k^{-\vartheta}}{k^{\gamma}+x^{\gamma}} .
$$

Then $S: \mathbb{R}_{0}^{+} \rightarrow \mathbb{R}^{+}$is continuous and for $\gamma>0$ even strictly decreasing. As for the asymptotic behavior of $S$, let $x_{0}>0$ be arbitrary. Then there exist positive constants $C_{1}$ and $C_{2}$ such that for all $x \geqslant x_{0}$ we have

$$
S(x) \in\left[C_{1}, C_{2}\right] \cdot \begin{cases}x^{-\gamma} & \text { for } \vartheta>1 \\ x^{-\gamma} \cdot \ln (1+x) & \text { for } \vartheta=1 \\ x^{1-\vartheta-\gamma} & \text { for } \vartheta<1 .\end{cases}
$$

Proof. Standard results on series of functions imply the continuity of $S$ on the closed interval $[0, \infty)$, as well as its differentiability on $(0, \infty)$. The derivative is obtained by term-wise differentiation and is always negative. For $\gamma=0$ there is nothing to show, as then $\vartheta>1$ and $S$ is a constant.

As for the asymptotic behavior of $S$, assume first that $\vartheta>1$, and consider the function $x^{\gamma} \cdot S(x)=\sum_{k \in \mathbb{N}} k^{-\vartheta} x^{\gamma} /\left(k^{\gamma}+x^{\gamma}\right)$. The derivative of this function can also be computed by term-wise differentiation, and this immediately implies that $x^{\gamma} \cdot S(x)$ is increasing. Together with $x^{\gamma} S(x) \rightarrow \sum_{k \in \mathbb{N}} k^{-\vartheta}<\infty$ for $x \rightarrow \infty$, this establishes the asymptotic behavior of $S$.

The case $0 \leqslant \vartheta \leqslant 1$ is more complicated due to the divergence of $\sum_{k \in \mathbb{N}} k^{-\vartheta}$. Using the fact that the terms of the series defining $S$ are decreasing with respect to $k$, a standard Riemann sum argument furnishes $f(x) \leqslant S(x) \leqslant f(x)+1 /\left(1+x^{\gamma}\right)$, where

$$
f(x)=\int_{1}^{\infty} \frac{k^{-\vartheta}}{k^{\gamma}+x^{\gamma}} \mathrm{d} k=x^{1-\vartheta-\gamma} \cdot \int_{1 / x}^{\infty} \frac{k^{-\vartheta}}{k^{\gamma}+1} \mathrm{~d} k .
$$

For $0 \leqslant \vartheta<1$ we have $\int_{0}^{\infty} k^{-\vartheta} /\left(k^{\gamma}+1\right) \mathrm{d} k<\infty$. Thus, there are $x$-independent positive constants $c$ and $C$ such that $c \leqslant x^{\gamma+\vartheta-1} \cdot f(x) \leqslant C$ for arbitrary $x \geqslant x_{0}$, and therefore $c x^{1-\gamma-\vartheta} \leqslant$ $S(x)$ for $x \geqslant x_{0}$. The upper bound follows from the estimate

$$
C x^{1-\vartheta-\gamma}+\frac{1}{1+x^{\gamma}}=x^{1-\vartheta-\gamma}\left(C+x^{\vartheta-1} \cdot \frac{x^{\gamma}}{1+x^{\gamma}}\right)=O\left(x^{1-\vartheta-\gamma}\right)
$$

for $x \rightarrow \infty$. The remaining case $\vartheta=1$ can be handled analogously. One only has to note that in this case $f(x)=\gamma^{-1} x^{-\gamma}\left(\ln \left(1+x^{\gamma}\right)\right)$.

In combination with (23) the above lemma allows us to determine $w_{L}^{2}(\infty)$. To this end, fix an arbitrary $L_{0}>0$. With $x=L^{d}$ and $\vartheta=2 m / d$, the continuity of $S$ on $\left[0,\left(L_{0}\right)^{d}\right]$ established in 
Lemma 5.2 implies for $L \in\left(0, L_{0}\right]$

$$
w_{L}^{2}(\infty) \in[c, C] \cdot L^{2 m-d(1-\gamma)} \cdot \sum_{k \in \mathbb{N}} \frac{k^{-2 m / d}}{k^{\gamma}+L^{d \gamma}} \subset[c, C] \cdot L^{2 m-d(1-\gamma)} .
$$

For $L \geqslant L_{0}$ we obtain

$$
w_{L}^{2}(\infty) \in[c, C] \cdot L^{2 m-d} \cdot \begin{cases}1 & \text { for } 2 m>d \\ \ln (1+L) & \text { for } 2 m=d \\ L^{d-2 m} & \text { for } 2 m<d\end{cases}
$$

Notice that the main problems in the proof of the previously applied lemma arise when $\vartheta=2 m / d \leqslant 1$. This corresponds to the case $2 m \leqslant d$, which ensures the non-existence of the mean interface width for the problem with space-time white noise.

The error estimate for $w_{L}^{2}(t)$ with respect to $w_{L}^{2}(\infty)$ is completely analogous to (15) and (16). The results derived in this section are presented collectively in Theorem 5.3 of Section 5.3.

\subsection{Transient behavior}

Next we consider small times $t>0$. This is accomplished similarly to the white noise case. We begin our derivations by noticing that the argument in the lower bound series in (5.1) is decreasing with respect to $k$, as well as continuous at $k=0$ with

$$
\lim _{k \rightarrow 0} \frac{1-\mathrm{e}^{-2 c_{A} k^{2 m / d} L^{-2 m} t}}{k^{2 m / d} \cdot\left(k^{\gamma} L^{-d \gamma}+1\right)} \cdot L^{2 m-d}=2 c_{A} t L^{-d} .
$$

An analogous statement holds for the argument in the upper bound series of (5.1). Together with a standard Riemann sum argument this implies the estimate

$$
\frac{c_{\Gamma}}{2 C_{A} C_{G}} \cdot\left(f_{1}(t)-2 c_{A} t L^{-d}\right) \leqslant w_{L}^{2}(t) \leqslant \frac{C_{\Gamma}}{2 c_{A} c_{G}} \cdot f_{2}(t),
$$

where

$$
\begin{aligned}
f_{1}(t) & :=L^{2 m-d+d \gamma} \cdot \int_{0}^{\infty} \frac{1-\mathrm{e}^{-2 c_{A} k^{2 m / d} L^{-2 m} t}}{k^{2 m / d} \cdot\left(k^{\gamma}+L^{d \gamma}\right)} \mathrm{d} k \\
& =t^{1-(1-\gamma) d /(2 m)} \cdot \int_{0}^{\infty} \underbrace{\frac{1-\mathrm{e}^{-2 c_{A} k^{2 m / d}}}{k^{2 m / d} \cdot\left(k^{\gamma}+t^{d \gamma /(2 m)}\right)}}_{=: I(k, t)} \mathrm{d} k .
\end{aligned}
$$

Here we used the substitution $\hat{k}=k t^{d /(2 m)} L^{-d}$. The definition of the upper bound $f_{2}(t)$ is similar to the one above-one just has to replace $c_{A}$ by $C_{A}$. In the following, we derive both upper and lower bounds for $f_{1}$, which immediately establishes similar bounds for $f_{2}$. Notice that for fixed $t>0$ the integrand $I$ in the definition of $f_{1}$ is always integrable, provided $\gamma+2 m / d>1$. For $t=0$ we have a singularity at $k \rightarrow 0$, which is not integrable if $\gamma \geqslant 1$. Moreover, the fact that $k^{-2 m / d}$ is not integrable on $(1, \infty)$ if $2 m \leqslant d$ complicates the discussion for large values of $t$. Therefore, we consider several cases: The integrals of $I(\cdot, t)$ over $(0,1)$ and $(1, \infty)$ will be considered separately. Furthermore, we distinguish between $t \leqslant t_{0}$ and $t \geqslant t_{0}$ for some arbitrary, but fixed $t_{0}>0$. 
Case A. $\quad t \leqslant t_{0}$ and $\int_{1}^{\infty} I(k, t) \mathrm{d} k$.

Applying the estimate $k^{\gamma} \leqslant k^{\gamma}+t^{\gamma d /(2 m)} \leqslant k^{\gamma}+t_{0}^{\gamma d /(2 m)}$ for all $0 \leqslant t \leqslant t_{0}$ to the denominator in $\int_{1}^{\infty} I(k, t) \mathrm{d} k$, this integral can be bounded by two $t$-independent integrals, whose convergence is a consequence of $\gamma+2 m / d>1$. Thus,

$$
\int_{1}^{\infty} I(k, t) \mathrm{d} k \in[c, C] \quad \text { for all } \quad 0 \leqslant t \leqslant t_{0}
$$

and two $t$-independent constants $0<c<C$.

Case B. $\quad t \geqslant t_{0}$ and $\int_{1}^{\infty} I(k, t) \mathrm{d} k$.

If $2 m>d$, then the integrability of $k^{-2 m / d}$ on $(1, \infty)$ allows us to multiply $I(k, t)$ by $t^{\gamma d /(2 m)}$, bound the term $t^{\gamma d /(2 m)} /\left(k^{\gamma}+t^{\gamma d /(2 m)}\right)$ by two $t$-independent constants as above, and finally bound $t^{\gamma d /(2 m)} \cdot \int_{1}^{\infty} I(k, t) \mathrm{d} k$. This furnishes

$$
t^{\gamma \cdot d /(2 m)} \cdot \int_{1}^{\infty} I(k, t) \mathrm{d} k \in[c, C] \quad \text { for all } \quad t \geqslant t_{0}, \quad \text { provided } 2 m>d .
$$

The preceding does not apply to the case $2 m \leqslant d$. Here we use

$$
\int_{1}^{\infty} I(k, t) \mathrm{d} k \in[c, C] \cdot \int_{1}^{\infty} \frac{k^{-2 m / d}}{k^{\gamma}+t^{\gamma d /(2 m)}} \mathrm{d} k
$$

in connection with the identity

$$
\int_{1}^{\infty} \frac{k^{-2 m / d}}{k^{\gamma}+t^{\gamma d /(2 m)}} \mathrm{d} k=t^{-1+(1-\gamma) d /(2 m)} \cdot \int_{t^{-d /(2 m)}}^{\infty} \frac{k^{-2 m / d}}{k^{\gamma}+1} \mathrm{~d} k .
$$

If $2 m<d$, then the integral in the right-hand side of this identity has a positive limit as $t \rightarrow \infty$, i.e. for $t \geqslant t_{0}$ it can be bounded by two $t$-independent constants $0<c<C$. This is no longer the case if $2 m=d$. However, in this case the integral can be evaluated explicitly and is given by $\ln \left(1+t^{\gamma}\right) / \gamma$. Altogether we obtain for arbitrary $t \geqslant t_{0}$ the estimates

$$
\int_{1}^{\infty} I(k, t) \mathrm{d} k \in[c, C] \cdot \begin{cases}t^{-\gamma \cdot d /(2 m)} & \text { for } 2 m>d \\ t^{-\gamma} \cdot \ln (1+t) & \text { for } 2 m=d \\ t^{-1+(1-\gamma) \cdot d /(2 m)} & \text { for } 2 m<d\end{cases}
$$

with $t$-independent constants $0<c<C$.

Case C. $\quad t \leqslant t_{0}$ and $\int_{0}^{1} I(k, t) \mathrm{d} k$.

Recalling that the function $\left(1-\mathrm{e}^{-2 c_{A} k^{2 m / d}}\right) \cdot k^{-2 m / d}$ has the positive limit $2 c_{A}$ as $k \rightarrow 0$, we can bound the corresponding term in the definition of $I(k, t)$ above and below by positive constants. Furthermore, it can easily be verified that for every $q \geqslant 0$ there exist positive constants $0<c_{q}<C_{q}$ such that for all $a, b \geqslant 0$ the inequality $c_{q} \cdot(a+b)^{q} \leqslant a^{q}+b^{q} \leqslant C_{q} \cdot(a+b)^{q}$ holds. This furnishes

$$
\int_{0}^{1} I(k, t) \mathrm{d} k \in[c, C] \cdot \int_{0}^{1}\left(k+t^{d /(2 m)}\right)^{-\gamma} \mathrm{d} k .
$$


Obviously, we have

$$
\int_{0}^{1}\left(k+t^{d /(2 m)}\right)^{-\gamma} \mathrm{d} k= \begin{cases}\frac{\left(1+t^{d /(2 m)}\right)^{1-\gamma}-t^{(1-\gamma) \cdot d /(2 m)}}{1-\gamma} & \text { for } \quad \gamma \neq 1 \\ \ln \left(1+t^{-d /(2 m)}\right) & \text { for } \quad \gamma=1\end{cases}
$$

which immediately implies

$$
\int_{0}^{1} I(k, t) \mathrm{d} k \in[c, C] \cdot \begin{cases}1 & \text { for } 0 \leqslant \gamma<1 \\ \ln \left(1+t^{-1}\right) & \text { for } \gamma=1 \\ t^{(1-\gamma) \cdot d /(2 m)} & \text { for } \gamma>1\end{cases}
$$

for all $t \leqslant t_{0}$, with two $t$-independent constants $0<c<C$.

Case D. $t \geqslant t_{0}$ and $\int_{0}^{1} I(k, t) \mathrm{d} k$.

Also in this case the formulas given in (28) and (29) remain valid. Mean value theorem type arguments now yield

$$
\int_{0}^{1} I(k, t) \mathrm{d} k \in[c, C] \cdot t^{-\gamma \cdot d /(2 m)} \quad \text { for all } \quad t \geqslant t_{0}
$$

with two $t$-independent constants $0<c<C$.

Combining the estimates in (26), (27), (30), and (31) with the identity in (25), we finally obtain the following description of the asymptotic behavior of $f_{i}(t)$, where $i=1,2$ :

$$
f_{i}(t) \in[c, C] \cdot\left\{\begin{array}{ll}
t^{1-d /(2 m)} & \text { for } 2 m>d \\
\ln (1+t) & \text { for } 2 m=d \\
1 & \text { for } 2 m<d
\end{array} \text { and } t \geqslant t_{0},\right.
$$

as well as

$$
f_{i}(t) \in[c, C] \cdot\left\{\begin{array}{ll}
t^{1-(1-\gamma) \cdot d /(2 m)} & \text { for } 0 \leqslant \gamma<1 \\
t \cdot \ln \left(1+t^{-1}\right) & \text { for } \gamma=1 \\
t & \text { for } \gamma>1
\end{array} \text { and } t \leqslant t_{0} .\right.
$$

Error bounds can be obtained analogous to the space-time white noise case using (24). In the present situation, the absolute error $\left|C_{e} \cdot f_{1}(t)-w_{L}^{2}(t)\right|$, with $C_{e}=c_{\Gamma} /\left(2 C_{A} C_{G}\right)$ as in (24), is bounded by $C t L^{-d}$, where $C$ is independent of $t$ and $L$. As for the relative error $\left|C_{e} \cdot f_{1}(t)-w_{L}^{2}(t)\right| /\left(C_{e} \cdot f_{1}(t)\right)$ we have to consider different cases. The above formulas imply

$$
\left|\frac{C_{e} \cdot f_{1}(t)-w_{L}^{2}(t)}{C_{e} \cdot f_{1}(t)}\right| \leqslant C \cdot L^{-d} \cdot\left\{\begin{array}{ll}
t^{d /(2 m)} & \text { for } 2 m>d \\
\frac{t}{\ln (1+t)} & \text { for } 2 m=d \\
t & \text { for } 2 m<d
\end{array} \text { and } t \geqslant t_{0},\right.
$$

as well as

$$
\left|\frac{C_{e} \cdot f_{1}(t)-w_{L}^{2}(t)}{C_{e} \cdot f_{1}(t)}\right| \leqslant C \cdot L^{-d} \cdot\left\{\begin{array}{ll}
t^{(1-\gamma) \cdot d /(2 m)} & \text { for } 0 \leqslant \gamma<1 \\
\frac{1}{\ln \left(1+t^{-1}\right)} & \text { for } \gamma=1 \\
1 & \text { for } \gamma>1
\end{array} \text { and } t \leqslant t_{0} .\right.
$$


Using these error bounds one can now easily calculate $t$-intervals on which the behavior of the mean interface width is described by the functions $f_{1}(t)$ and $f_{2}(t)$ up to some prespecified error $p>0$. The results are presented in Theorem 5.4 in the next section.

\subsection{Main results and discussion}

In this section we collect the results describing the evolution of the mean interface width $w_{L}(t)$ which were derived in the previous two sections. As for the long-time behavior, we proved the following results in Section 5.1.

TheOREm 5.3 Suppose that Assumptions 4.1, 4.3, and 5.1 are satisfied with $n=m / d$. Furthermore, assume $\gamma+2 m / d>1$ for the existence of $W_{A}$ in $L^{2}(\Omega, X)$.

Then for arbitrary $L_{0}>0$ there exist positive constants $C_{1}<C_{2}$ which are independent of $t$ and $L$ such that the following holds. For arbitrary $p>0$, there exists a constant $C_{p}>0$ with

$$
w_{L}^{2}(t) \in[1-p, 1] \cdot w_{L}^{2}(\infty) \text { for all } t \geqslant C_{p} \cdot L^{2 m} .
$$

As for $w_{L}^{2}(\infty)$ we have

$$
w_{L}^{2}(\infty) \in\left[C_{1}, C_{2}\right] \cdot L^{2 m-(1-\gamma) d} \quad \text { for } \quad 0<L \leqslant L_{0}
$$

and

$$
w_{L}^{2}(\infty) \in\left[C_{1}, C_{2}\right] \cdot\left\{\begin{array}{ll}
L^{2 m-d} & \text { for } 2 m>d \\
\ln (1+L) & \text { for } 2 m=d \\
1 & \text { for } 2 m<d
\end{array} \text { and } L \geqslant L_{0} .\right.
$$

Note that $2 m>d$ implies the existence of the mean interface width for the equation with spacetime white noise. For small domains the colored noise alters the long-time behavior by a factor $L^{d \gamma}$. For large domains the regularity of the noise does not change the long-time behavior in the case when there are solutions for the equation with space-time white noise. An important case is $2 m=d$. Here, the roughness of a surface corresponding to space-time white noise is not defined, but for colored noise, we get a logarithmic growth of $w_{L}^{2}(\infty)$ for $L \rightarrow \infty$. This is discussed further in Remark 5.6.

The transient behavior was the subject of Section 5.2. The results obtained there can be summarized as follows.

TheOREm 5.4 Suppose that Assumptions 4.1, 4.3, and 5.1 are satisfied with $n=m / d$. Furthermore, assume $\gamma+2 m / d>1$ for the existence of $W_{A}$ in $L^{2}(\Omega, X)$.

Then for arbitrary but fixed $t_{0}>0$ there exist $t$ - and $L$-independent positive constants $C_{1}, \ldots, C_{4}$ such that the following is true. For given $0<p \ll 1$ there exist positive constants which are all denoted by $C_{p}$ and independent of $t$ and $L$, such that for $t \geqslant t_{0}$ we have

$$
w_{L}^{2}(t) \in\left[C_{1}-p, C_{2}\right] \cdot\left\{\begin{array}{lll}
t^{1-d /(2 m)} & \text { for } 2 m>d, & t \leqslant C_{p} \cdot L^{2 m} \\
\ln (1+t) & \text { for } 2 m=d, & t / \ln (1+t) \leqslant C_{p} \cdot L^{d} \\
1 & \text { for } 2 m<d, & t \leqslant C_{p} \cdot L^{d}
\end{array}\right.
$$


as well as for $t \leqslant t_{0}$

$$
w_{L}^{2}(t) \in\left[C_{3}-p, C_{4}\right] \cdot\left\{\begin{array}{lll}
t^{1-(1-\gamma) \cdot d /(2 m)} & \text { for } 0 \leqslant \gamma<1, & t \leqslant C_{p} \cdot L^{2 m /(1-\gamma)} \\
t \cdot \ln \left(1+t^{-1}\right) & \text { for } \gamma=1, & t \leqslant 1 /\left(\mathrm{e}^{C_{p} / L^{d}}-1\right) \\
t & \text { for } \gamma>1, & L \leqslant C_{p} .
\end{array}\right.
$$

Combining Theorem 5.3 and 5.4 we obtain the following remarks.

REMARK 5.5 For large domain size $L$ and for large times $t \geqslant t_{0}$ one obtains roughly the same behavior of $w_{L}(t)$ for both space-time white and colored noise, in the case when both solutions exist, i.e. $2 m>d$. Here, the exponents $\alpha=m-d / 2$ and $z=2 m$ are well defined. Only for $t \leqslant t_{0}$ we observe a change in the behavior of $w_{L}(t)$ due to regularity. Therefore, we cannot define one exponent $\beta$.

Nevertheless, we obtain for $t \geqslant t_{0}$ that $\beta=1 / 2-d /(4 m)$, which is the exponent corresponding to space-time white noise. For $t \leqslant t_{0}$ and $\gamma \in[0,1)$ we have $\beta=1 / 2-(1-\gamma) \cdot d /(4 m)$. Apart from the different behavior for $t \leqslant t_{0}$, this was conjectured by a rescaling argument in [1].

REMARK 5.6 An important case for dimension $d=2$ is $2 m=d$. This corresponds, for example, to a problem where $A$ is given by $-\Delta_{\text {per }}$ on a rectangular domain. Here, the roughness of a surface corresponding to space-time white noise is not defined, but for colored noise with $\gamma>0$, we observe logarithmic behavior, and the constants $\alpha$ and $\beta$ are not well defined. More precisely, we have $w_{L}^{2}(\infty) \in[c, C] \cdot \ln (1+L)$ for $L \geqslant L_{0}$, as well as $w_{L}^{2}(t) \in[c, C] \cdot \ln (1+t)$ for $t_{0} \leqslant t$, but only up to times $t / \ln (1+t) \leqslant C_{p} \cdot L^{2 m}$. For $t \leqslant t_{0}$ and $\gamma \in(0,1)$ the mean interface width grows like $t^{\gamma / 2}$.

We already mentioned earlier that it is also possible to consider more general types of colored noise. One such example is colored noise for which the upper and lower bounds on the coefficients $\alpha_{k}(L)$ exhibit different algebraic decay rates. To illustrate this, we focus briefly on the situation $2 m>d$, large domain size $L$, and algebraic decay rates $\gamma$ smaller than 1 , which was discussed in Remark 5.5.

Corollary 5.7 Assume that $A$, and $G$ are given as in Theorems 5.3 or 5.4. Furthermore, suppose that $W$ is a $Q$-Wiener process as in Assumption 5.1 where (21) has been replaced by

$$
\frac{c_{\Gamma}}{1+k^{\gamma_{1}} \cdot L^{-d \gamma_{1}}}<\alpha_{k}^{2}(L)<\frac{C_{\Gamma}}{1+k^{\gamma_{2}} \cdot L^{-d \gamma_{2}}}
$$

for some constants $0 \leqslant \gamma_{2} \leqslant \gamma_{1}<1$. Suppose $2 m>d$ in order to guarantee the existence of a solution to the space-time white noise problem in $L^{2}(\Omega, X)$.

Then for given constants $L_{0}>0$ and $t_{0}>0$ there exist $t$ - and $L$-independent constants $C_{1}, \ldots, C_{6}$ such that the following is true. For fixed $0<p \ll 1$ there exist positive constants $c_{p} \leqslant C_{p}$ (cf. Fig. 4) and $C_{p}^{\prime}$ independent of $t$ and $L$ such that for $L \geqslant L_{0}$ and $L \geqslant\left(t_{0} / C_{p}^{\prime}\right)^{\left(1-\gamma_{i}\right) /(2 m)}$ for $i \in\{1,2\}$ we have

$$
\begin{aligned}
& \left(C_{1}-p\right) \cdot t^{1-\left(1-\gamma_{1}\right) \cdot d /(2 m)} \leqslant w_{L}^{2}(t) \leqslant C_{2} \cdot t^{1-\left(1-\gamma_{2}\right) \cdot d /(2 m)} \quad \text { for } t \leqslant t_{0}, \\
& \left(C_{3}-p\right) \cdot t^{1-d /(2 m)} \leqslant w_{L}^{2}(t) \leqslant C_{4} \cdot t^{1-d /(2 m)} \quad \text { for } \quad t_{0} \leqslant t \leqslant c_{p} \cdot L^{2 m}
\end{aligned}
$$




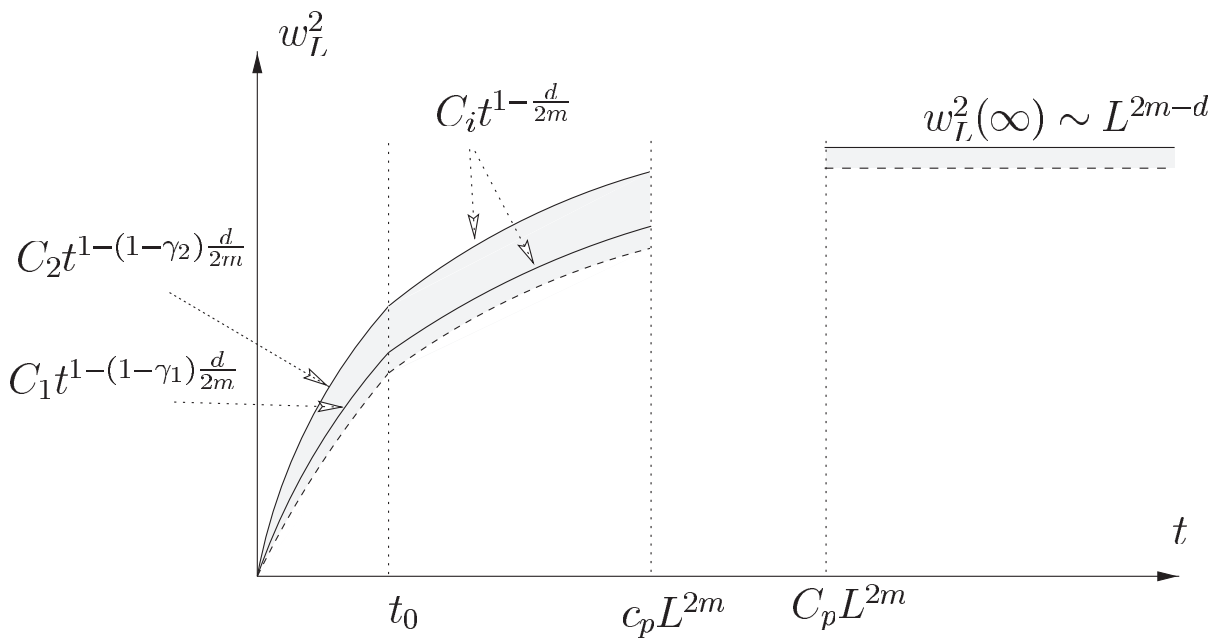

FIG. 4. Surface roughness for colored noise with relative error $p>0$ for sufficiently large domains and slightly colored noise provided the space-time white noise solution exists.

where the last case is not empty only if $L \geqslant\left(t_{0} / c_{p}\right)^{1 /(2 m)}$. Moreover,

$$
w_{L}^{2}(t) \in[1-p, 1] \cdot w_{L}^{2}(\infty) \text { for } t \geqslant C_{p} \cdot L^{2 m},
$$

where

$$
w_{L}^{2}(\infty) \in\left[C_{5}, C_{6}\right] \cdot L^{2 m-d} .
$$

Notice that one obtains different asymptotic bounds on the behavior of the mean interface width for $t \leqslant t_{0}$. Despite this fact, for $t \geqslant t_{0}$ the behavior is similar to the white noise case, up to the constants. The result of the above corollary is sketched in Fig. 4.

\section{Acknowledgements}

The work of SM-P was supported by the Deutsche Forschungsgemeinschaft, 'Heisenbergstipendium', Ma 1583/3-1.

\section{REFERENCES}

1. Barabasi, A. \& Stanley, H. Fractal Concepts in Surface Growth. Cambridge University Press, (1995).

2. BlÖMKER, D. Stochastic partial differential equations and surface growth, PhD Thesis, Universität Augsburg, (2000).

3. BlÖMKer, D., MAiER-PAAPE, S., \& WANNer, T. Surface roughness in molecular beam epitaxy. Stochastics Dynam. 1, (2001) 239-260.

4. Courant, R. \& Hilbert, D. Methods of Mathematical Physics. Intersciences, New York (1953).

5. DA Prato, G. \& ZAbczyK, J. Stochastic equations in infinite dimensions. In Encyclopedia of Mathematics and its Application 44. Cambridge University Press, (1992).

6. Edwards, S. \& Wilkinson, D. The surface statistics of a granular aggregate. Proc. R. Soc. London, Series A 381, (1982) 17-31. 
7. FAmily, F. \& VicSEK, T. Scaling of the active zone in the Eden process on percolation networks and the ballistic deposition model. J. Phys. A 18, (1985) L75-L81.

8. HALPIN-HEALY, T. \& ZHANG, Y. Kinetic roughening phenomena, stochastic growth, directed polymers and all that. Phys. Rep. 254, (1995) 215-414.

9. Hunt, A., Sander, L., Orme, C., Orr, B., Johnson, M., Graff, D., \& Sudijono, J. Stable and unstable growth in MBE. Phys. Rev. Lett. 72, (1994) 116-119.

10. Hunt, A., Sander, L., Orme, C., OrR, B., \& Williams, D. Instabilities in MBE growth. Europhys. Lett. 27, (1994) 611-616.

11. Kardar, M., PARISI, G., \& Zhang, Y.-C. Dynamic scaling of a granular aggregate. Phys. Rev. Lett. 56, (1986) 889-892.

12. Krug, J. Origins of scale invariance in growth processes. Adv. Phys. 46, (1997) 139-282.

13. KRUG, J. \& SpOHN, H. Kinetic roughening of growing surfaces. In: Godrèche, C. (ed), Solids far from Equilibrium. pp. 479-582. Cambridge University Press, (1991).

14. Mandelbrot, B. Fractals in Physics. Elsevier, Amsterdam (1986).

15. Marsili, M., Maritan, A., Toigo, F., \& Banavar, J. Stochastic growth equations and reparametrization invariance. Rev. Mod. Phys. 68, (1996) 963-983.

16. Meakin, P. The growth of rough surfaces and interfaces. Phys. Rep. 235, (1993) 189-289.

17. Medina, E., Hwa, T., KadAR, M., \& Zhang, Y. Burgers equation with correlated noise: renormalization-group analysis and applications to directed polymers and interface growth. Phys. Rev. A 39, (1989) 3053-3075.

18. Raible, M., Mayr, S. G., Linz, S. J., Moske, M., Hänggi, P., \& Samwer, K. Amorphous thin film growth: theory compared with experiment. Europhys. Lett. 50, (2000) 61-67.

19. Sigert, M. \& PlischKe, M. Slope selection and coarsening in molecular beam epitaxy. Phys. Rev. Lett. 73, (1994) 1517-1520.

20. Wolf, D. \& Villain, J. Growth with surface diffusion. Europhys. Lett. 13, (1990) 389-394. 